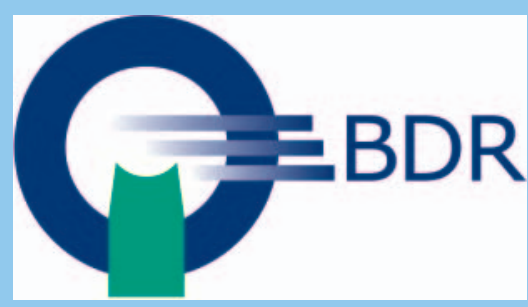

\title{
UEMS - Das Haus, auch der Radiologie, in Europa
}

Auch in diesem Jahr haben BDR und DRG wieder aktiv an den Sitzungen der für uns zuständigen europäischen Gremien im Rahmen der UEMS (Union Européenne des Médecins Spécialistes) teilgenommen. Die Sitzung der UEMS Section of Radiology fand dabei einmal mehr Anfang Oktober in Rom, die Herbstsitzung des Europa-Ausschusses des SpiFa (Spitzenverband Fachärzte Deutschlands) wenige Tage später in Berlin statt.

Das Treffen der UEMS Section of Radiology in der Poliklinik der Universität von Rom am 06.10.2017 stand im Zeichen der Konsolidierung und der Vorbereitung kommender Meetings der UEMS. Insbesondere die jährliche Council-Sitzung als oberstes Entscheidungsgremium der UEMS, die wenige Tage später in Brüssel stattfand, wurde vordiskutiert.

Seit der Übernahme des Vorsitzes durch Prof. Paolo Ricci (Rom) im Frühjahr 2016 wurden die Aktivitäten der Sektion erheblich ausgeweitet. So ist die Section of Radiology aktuell eine der aktivsten Sektionen der UEMS. Seinen Höhepunkt fanden diese mit der Fertigstellung und Vorlage des European Diploma of Radiology (EDir) beim diesjährigen Council-Meeting. Gemeinsam mit der European Society of Radiology (ESR) war dazu in den letzten Jahren ein Curriculum nach den Regeln der European Training Requirements (ETR) für einen „Europäischen Facharzt“ erarbeitet worden. Für alle Staaten, in denen nationale Regularien zur Erlangung des radiologischen Facharztes bestehen, gilt dabei, dass das EDir nur zusätzlich und nicht anstatt der nationalen Facharztanerkennung erworben werden kann.

Weiterhin erfreulich stellt sich die finanzielle Situation der Sektion dar. So konnten trotz einer Reduktion der Mitgliedsbeiträge alle Aufgaben vollumfänglich erfüllt werden, die finanzielle Ausstattung der Sektion ist stabil.

Die Zusammenarbeit zwischen der Section of Radiology der UEMS und der ESR (European Society of Radiology) hat sich auch im vergangenen Jahr weiter stabilisiert. So war auch in diesem Jahr der aktuelle ESRPräsident Prof. Derchi zur Sektionssitzung gekommen. Für den kommenden ECR ist, wie in den vergangenen Jahren, wieder eine gemeinsame Sitzung von UEMS (Section of Radiology) und der ESR geplant. Als besonders fruchtbar erweist sich weiterhin das gemeinsame Sekretariat von ESR und UEMS als ständige Vertretung der Radiologie in Brüssel. Es ist im sogenannten Domus Medica in der Rue de l'Industrie in Brüssel, in unmittelbarer Nähe zu den europäischen Institutionen, untergebracht.

Die Herbstsitzung des Europa-Ausschusses des SpiFa am 10.10.2018 in Berlin stand zunächst im Zeichen der Neuwahl des Vorstandes. Einstimmig wurde dabei als Nachfolger von Herrn Dr. Ulrich, der in der Frühjahrssitzung zurückgetreten war, Herr Dr. Klaus König, Vertreter des Berufsverbands der Frauenärzte (BVF), gewählt. Zu seinem Nachfolger wählte der Ausschuss, ebenfalls einstimmig, Herrn Dr. Klaus Bodmann, Vertreter des Berufsverbands der Internisten (BDI).

Im Gegensatz zur Section of Radiology der UEMS ist die finanzielle Situation des Europa-Ausschusses des SpiFa deutlich angespannt. Um weiterhin den Aufgaben gerecht zu werden, wurde eine, vorher innerhalb der Verbände abgesprochene, Erhöhung der Beiträge in der Herbstsitzung am 10.10.2018 in Berlin beschlossen. Von Seiten des Vorstands des Europa-Ausschusses war dabei besonders auf die Notwendigkeit einer ständigen

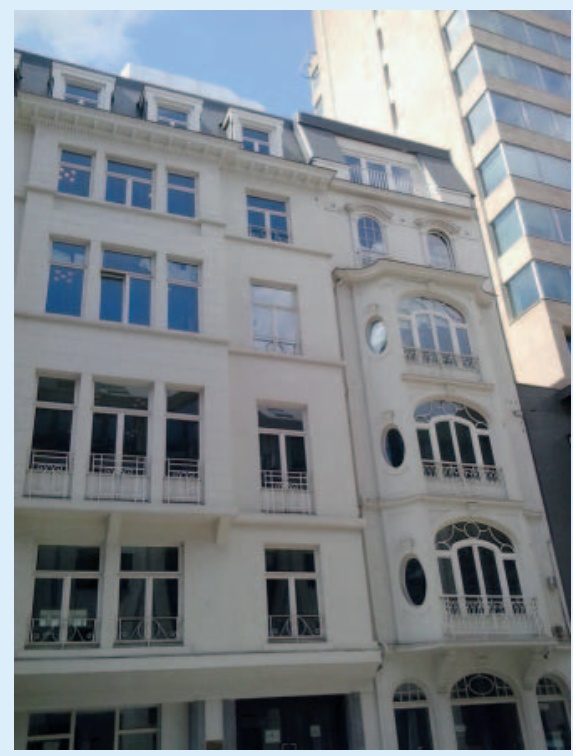

Domus Medica in Brüssel (Copyright UEMS).

Vertretung der deutschen Fachärzte in Brüssel hingewiesen worden. Derzeit finden die deutschen Fachärzte temporär Unterschlupf in den Räumen der von-Beust-Beratungsgesellschaft in Brüssel. Die Notwendigkeit einer ständigen Vertretung für eine effektive $\mathrm{Ar}$ beit innerhalb der UEMS wurde, nicht zuletzt vom BDR aus den Erfahrungen der Radiologie, in der Diskussion vertreten.

Auch die Sitzung des Europa-Ausschusses des SpiFa diente der Vorbereitung der Council-Sitzung der UEMS wenige Tage später. Nach fachlicher Darstellung durch den BDR beauftragte der Ausschuss einstimmig seinen Vertreter bei der Council-Sitzung für eine Annahme des EDiR zu stimmen. Besonders wichtig war den Teilnehmern dabei erneut die durch das EDiR nicht angetastete Hoheit der nationalen Institutionen zur Verleihung des radiologischen Facharztes - in Deutschland durch die Landesärztekammern. 
Aus radiologischer Sicht war die Verabschiedung des EDiR in der Council-Sitzung der UEMS im Herbst 2018 in Brüssel ein wichtiger Meilenstein europäischer Berufspolitik. Nicht nur, weil das Projekt von europäischer Fachgesellschaft (ESR) und Section of Radiology der UEMS gemeinsam und im Einvernehmen erarbeitet worden war. Auch weil es frühzeitig gelungen ist, im Vergleich zu anderen Fachdisziplinen, eine europäische Ergänzung zu den nationalen Fachärz- ten zu schaffen, ohne die nationalen Kompetenzen zu beschneiden. Das Interesse an EDiR unter den jungen Facharztanwärterinnen und -anwärtern zeigt, dass bei zunehmend mobiler und internationaler Gesellschaft der Bedarf nach einer standardisierten europäischen Dokumentation erworbener Fachkenntnisse besteht. Der weite Weg durch die Institutionen bis zu einer europaweiten Anerkennung macht eine permanente Vertretung am Puls europä- ischer Politik unverzichtbar. Die Radiologie ist schon da, hat ihren festen Platz in Brüssel und der BDR ist dabei.

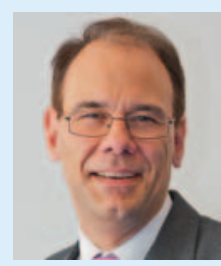

Prof. Hermann Helmberger, München 